\title{
Raymonde Monnier (dir.), Citoyen et citoyenneté sous la Révolution française
}

Actes du colloque international de Vizille des 24 et 25 septembre 2004, Paris, Société des études robespierristes, 2006, 310 p., ISBN

2-908327-54-6, $29 €$.

Melvin Edelstein

\section{(2) OpenEdition}

1 Journals

Édition électronique

URL : https://journals.openedition.org/ahrf/11297

DOI : 10.4000/ahrf. 11297

ISSN : 1952-403X

\section{Éditeur :}

Armand Colin, Société des études robespierristes

\section{Édition imprimée}

Date de publication : 1 septembre 2007

Pagination : 219-221

ISSN : 0003-4436

\section{Référence électronique}

Melvin Edelstein, «Raymonde Monnier (dir.), Citoyen et citoyenneté sous la Révolution française »,

Annales historiques de la Révolution française [En ligne], 349 | juillet-septembre 2007, mis en ligne le 29 décembre 2009, consulté le 01 juillet 2021. URL : http://journals.openedition.org/ahrf/11297 ; DOI : https://doi.org/10.4000/ahrf.11297 
Mot, syntagme, lexie, expression, notion, concept, idée, formule configurant l'idée de bonheur, voilà un enscmble de critères méthodologiques souvent difficile à manier. Les instruments proposés, les plus simples, nous aident à s'y retrouver en leur sein, avant même le recours à des méthodes plus complexes, la lexicométrie d'un côté, l'analyse de discours de l'autre. Souhaitons donc que cette initiative, qui va se préciser dans deux autres volumes, repose les bases d'avancées méthodologiques déjà éprouvées, mais qui ne sont pas passées dans l'usage commun des historiens, historiens des idées inclus.

Jacques GUILHAUMOU

Raymonde MONNIER (dir.), Citoyen et citoyenneté sous la Révolution française, actes du colloque international de Vizille des 24 et 25 septembre 2004, Paris, Société des études robespierristes, 2006, 310 p., ISBN 2-908327-54-6, $29 €$.

Les tensions du monde actuel conduisent à s'interroger sur les questions d'identité nationale et d'intégration des étrangers dans l'État-nation, sur les critères de la citoyenneté et l'accès au droit de la cité. Les historiens de la Révolution française sont au cœur de ces débats depuis l'époque du bicentenaire. Ainsi, cet ouvrage s'inscrit dans le droit fil de The French Revolution and the Meaning of Citizenship, paru en 1993, et du numéro 3 des Études révolutionnaires de la SER : Suffrage, citoyenneté et révolutions, 1789-1848, paru en 2002. En l'occurrence, l'ouvrage rassemble les communications présentées à l'occasion du colloque international qui a eu lieu en septembre 2004 au musée de la Révolution française de Vizille. En revenant à la fondation révolutionnaire du concept moderne de citoyenneté, ces approches plurielles d'historiens, de philosophes et de juristes permettent une meilleure compréhension de l'héritage révolutionnaire de la citoyenneté.

En introduction de l'ouvrage, Michel Pertuć contribuc à poser quelques problématiques majeures : " le modèle français appartient-il au passé ? " «Le modèle révolutionnaire de la citoyenneté a-t-il encore une pertinence ? "Nous trouverons peut-êtrc quelques réponses dans ces communications. Dans son rapport introductif, Bronislaw Baczko cite Chateaubriand pour démontrer comment le mot «citoyen » a remplacé celui de «Monsieur » pendant la décennie révolutionnaire ; tout un symbole!

Dans la première partie, intitulée « Notion et représentation », Luc Vincenti aborde les rapports entre les notions de citoyenneté et de démocratic dans la pensée de Rousseau. Si la conception rousseauiste de la citoyenneté nous conduit vers la démocratie, le philosophe n'est pas démocrate au sens strict ; il est moins encore totalitaire, même si l'individu doit se soumettre à la volonté générale. Jean Bart rappelle les rapports entrc citoyenneté et naturalité, ou nationalité. Pendant la décennie révolutionnaire, c'est la reconnaissance des droits politiques, c'est-à-dire de la citoyenneté, qui détermine la qualité de Français. En revanche, à partir du Consulat et depuis, c'est plutôt la nationalité qui fait d'un individu un Français et éventuellement un citoyen. En abordant une histoire comparée des questions juridiques, Matijn Van der Burg analyse l'impact du Code Napoléon sur le code civil hollandais. Il montre comment la codification du droit civil s'est adaptée aux mours nationales, notamment en matière de citoyenneté. Les juristes hollandais estiment que la distinction entre la citoyenneté et la qualité de Français est indési- 
rable. En étudiant les discours et adresses, les chansons et les images dans la République de Mayence à partir de 1792, Rolf Reichardt observe une évolution sémantique du mot Burger, parallèle à ce que s'est passé en France avec le mot citoyen. Patrice Higonnet aborde l'évolution historique des droits et des identités des citoyens dans les États-Unis et la France. Aux États-Unis, tout tournait autour de l'ethnicité. En France, le point de départ est l'universalisme. En revanche, l'État français refuse tout particularisme. Ensuite, Philippe Bourdin traite le citoyen dans « tous ses états littéraires ».

La deuxième partie, intitulée "Territoire et appartenances ", regroupe quatre communications. Celle de Daniel Nordman développe les innovations de la Révolution dans la construction territoriale et les nouvelles valeurs attachées au territoire. Puisant dans les dictionnaires du XVIII ${ }^{\circ}$ siècle, Raymonde Monnier met en lumière l'émergence de la notion de citoyen. Elle souligne l'importance des théories de l'utilité publique et la figure du citoyen contribuable dans l'avènement de la notion. Le problème du conflit entre le discours universaliste et les pratiques xénophobes est abordé par Anna Maria Rao au travers des réfugiés italiens et de l'expérience de la République Cisalpine. D'un côté, les patriotes italiens exilés en Cisalpine furent appelés « citoyens sans patrie " puisqu'ils n'avaient pas de territoire où exercer leurs droits ; d'un autre côté, les Italiens exilés en France furent considérés citoyens de tout pays libre puisque c'était la liberté qui faisait la nation. Lexpérience politique des patriotes italiens révèle les persistances d'une conception de la citoyenneté cosmopolite ; en même temps que les changements de cette conception face à l'émergence de l'État-nation et les possibilités d'une coexistence entre les deux. S'intéressant aux minorités en révolution, Christine Peyrard affirme que la Révolution a donné naissance au sens politique de ce terme, alors même que la souveraineté de la nation semble exclure les dissonances politiques.

La troisième partie, intitulée "Suffrage ", comprend six textes, essentiellement consacrés aux élections. S'interrogeant sur la pénurie d'études sur les élections révolutionnaires avant les annécs 1990, Serge Aberdam remarque comment l'article méthodologique de Jean-René Suratteau paru en 1968 a détourné les historiens de l'étude des élections révolutionnaires. Il retrace ensuite le parcours historiographique du sujet de la fin du XIX" siècle jusqu'à présent. Pour mettre fin au divorce entre l'historiographie des élections révolutionnaires et la construction d'une nouvelle historiographie politique, il fait appel a une synthèse sur les votes révolutionnaires. Malcolm Crook, quant à lui, met en valeur les assemblées électorales sous la Révolution comme une école où les participants ont fait leur apprentissage de la citoyenneté. Alors que Crook souligne le rôle joué par les assemblées électorales dans l'acculturation politique des citoyens, Philippe Tanchoux met l'accent sur les aspects négatifs des pratiques électorales. Tout en considérant la Révolution comme la transition entre les désignations communautaires d'Ancien Régime et le vote individuel, il affirme que cette avancée reste limitée. Le vote en assemblée ne préserve guère la volonté de chacun alors même que des pratiques communautaires réapparaissent, l'individualisation de la participation des électeurs n'étant menée à terme qu'en 1913 avec l'isoloir. Alors que les féministes dénoncent l'exclusion révolutionnaire des femmes de l'espace public, Élisabeth Sledziewski essaie de comprendre comment cette exclusion a été historiquement et idéologiquement possible. Elle affirme qu'on ne peut correctement rendre compte de cette exclusion civique des femmes si l'on oublie l'émancipation civile. Philippe Grateau montre comment les cahiers de doléances rédigés à l'occasion des États généraux 
peuvent enrichir notre connaissance de la construction de la citoyenneté. Ils constituent une expérience de démocratie " participative " où tous ceux qui le souhaitent s'associent au processus de délibération et de décision. S'adressant aux critiques de la culture politique révolutionnaire, qui nient sa modernité, Bernard Gainot lie les élections de la période du Directoire à la question du pluralisme politique. Affirmant que les historiens n'ont jamais été attentifs aux projets de code électoral, de droit d'association et de liberté de la presse en 1799, il trouve dans ces textes les fondements d'unc modernité politique. Les candidatures publiques sont de nouveau mises en avant. La loi doit protéger l'expression des minorités. Toutes vont dans le même sens : un pluralisme comme achèvement du libéralisme.

La quatrième partie, intitulée « Pratiques sociales », associe six communications. Jacques Poumarède rappelle comment les révolutionnaires ont repensé la famille à la lumière des nouveaux principes. La famille de l'ancien droit fabriquait des sujets ; la nouvclle famille régénérée devait désormais éduquer les citoyens. S'associant à la thèse d'E. Sledziewski, Christine Fauré argumente que l'offrande patriotique des femmes et filles d'artistes à l'Assemblée nationale le 7 septembre 1789 est une pratique alternative à l'exercice de la citoyenneté. Haim Burstin analyse le rapport entre travail et citoyenneté à Paris. Il constate que si, en général, l'accès à la citoyenneté se produit de facto par la politisation des revendications économiques, le combat pour l'extension de jure de la citoyenneté n'est pas l'apanage des travailleurs. La demande d'élargissement du suffrage ne semble pas être à l'origine une revendication du monde de travail. Si la question du travail rejoint celle de la citoyenneté, c'est la notion de la citoyenneté qui l'emporte en se superposant à celle du travail. Thomas Hippler aborde le rapport entre service militaire et citoyenneté. Il analyse d'emblée le rapport de la garde nationale à la citoyenneté, avant de porter l'attention sur la tension entre droits civiques et soumission disciplinaire au sein des armées. Dans un troisième temps, il s'agit d'évaluer quelles attitudes les soldats eux-mêmes avaient envers ces questions. Finalement, il met en valeur le renversement de causalité dans la relation entre citoyenneté et service militaire. Jean-Luc Chappey s'éloigne des historiens qui affirment que le propriétaire était la véritable pierre de touche du projet politique du Directoire. Sa réflexion s'appuie sur la prise en compte d'un critère « culturel » introduit dans la définition de la citoyenneté politique par la Constitution de l'an III ; se basant sur la pensée des Idéologues, il maintient que leur idéal fut une République des savants. Anne Simonin clôt cette partie en soulevant la question : comment un citoyen peut perdre ses droits sous la Révolution?

Invités à réexaminer la fondation révolutionnaire de la citoyenneté, ces auteurs présentent le sujet dans tous ses états. Ils mettent en lumière comment la conception de la citoyenneté était susceptible de différentes interprétations pendant la Révolution et par la suite. Les interrogations sur les théories et les pratiques de la citoyenneté révèlent la complexité et les contradictions du passage des principes aux actes. Si la France a fait son apprentissage de la citoyenneté pendant la Révolution, cet héritage complexe se révèle à la fois à l'origine du rassemblement national et des conflits actuels. 